\title{
Research on 4-UPUS Parallel Mechanism Ankle Joint Rehabil- itation Training Robot
}

\author{
Ao Hu${ }^{1}$, Qiang Yin ${ }^{2}$, Chengcai He ${ }^{3}$, Xiaohong Dun ${ }^{3}$ and Jia Ai ${ }^{1,3,}$, \\ 1 School of Mechanical and Electronic Engineering, Wuhan University of Technology, Wuhan \\ 430070,China; huao518@foxmail.com(A.H.); aijia@wru.edu.cn(J.A.) \\ 2 School of Mechanical Engineering,Wuhan Polytechnic University,Wuhan 430023,China; \\ yinqiang@whpu.edu.cn(Q.Y.) \\ 3 Wuhan Railway Vocational College Of Technology,Wuhan, 430205,China; \\ Hechengcai@wru.edu.cn(C.H.);Dunxiaohong@wru.edu.cn(X.D.); aijia@wru.edu.cn(J.A.) \\ * Correspondence: aijia@wru.edu.cn;
}

\begin{abstract}
In-depth research on the rehabilitation mechanism and motion characteristics of a rehabilitation training robot based on 4-UPUS parallel mechanism. The overall design and the working principle of each key component are explained, and the robot rehabilitation scheme is described. The static analysis and modal analysis of the robot bearing structure are carried out to verify whether the static mechanical characteristics of the mechanism meet the application requirements under working conditions.A simplified mathematical model of the six-bar linkage mechanism is established, and the kinematics of the mechanism is solved by the geometric analysis method. The motion characteristic diagram of each key point is obtained through simulation calculation. Establish the motion model of the parallel mechanism, and discuss the working space and motion performance analysis of the mechanism. The forward solution analysis of the mechanism position is carried out by using the numerical analysis method, and the three-dimensional graphics of the attitude angle and linear displacement of the reachable working space are obtained.Taking the UPUS single branch chain as the analysis object, the single open chain analysis method is used to solve the kinematics image of the corresponding surface in the working space of the mechanism, which verifies the correctness of the kinematics theoretical solution and the feasibility of simulation.The research results show that the 4-UPUS parallel mechanism rehabilitation training robot can cooperate with the ankle joint for rehabilitation training. It makes up for the single movement of the current lower limb rehabilitation robot and the unsatisfactory rehabilitation effect, and provides a reference for the practical application of the subsequent ankle joint rehabilitation robot system.
\end{abstract}

Keywords: Ankle joint rehabilitation robot Parallel mechanism Statics analysis Kinematics analysis Workspace

\section{Introduction}

The main research in this paper is that the 4-UPUS parallel mechanism rehabilitation training robot enables patients to receive systematic training in a variety of ways. It is mainly composed of a parallel mechanism composed of 4 UPUS, a six-bar linkage mechanism and a foot fixing device.It consists of two branches of the parallel mechanism that are responsible for the rehabilitation of the left and right ankle joints, including back extension, plantar flexion, valgus and varus. The internal rotation and external rotation of 
the ankle joint are completed by a six-bar linkage mechanism.By simulating the movement of the ankle joint, the drive motor pushes the mechanism to drive the ankle joint to an opening and closing angle during the movement. The circulatory rehabilitation action also makes the patient's ankle joint form neuromuscular memory, and the synergy of the various mechanisms greatly increases the chance of rehabilitation treatment.Rehabilitation robots can not only help patients with ankle joint injuries perform rehabilitation exercises, but also can be used for sports training for athletes and joint correction for the elderly. It can effectively improve the muscle strength of the ligaments around the ankle joint, and improve the flexibility, stability and coordination of the ankle joint.

It is composed of two Universal hinges (U), a hydraulic push rod Prismatic pair (P), and a Spherical pair $(S)$ in series, and a total of four kinematic chains are formed in parallel to form a 4-UPUS parallel mechanism.

It has good flexibility and athletic performance. The unique hinged varus and valgus training mode breaks the traditional mode and can effectively provide multi-modal training of the ankle joint, which can well complete plantar flexion and dorsiflexion. It has the advantages of dexterity, changeability and strong adaptability. The independent design of the two feet realizes the rehabilitation of the left and right ankles respectively, and at the same time adds a leg fixing device, which is more practical. The use of the six-link module can effectively overcome the lack of internal rotation and external rotation of the ankle joint in the traditional model. It can realize multiple degrees of freedom movement modes, so that the movement of each mechanism is more in line with the movement of the human ankle joint, and can provide reasonable rehabilitation training of the human ankle joint in complex postures.

The structure of this paper is as follows. Section 1 introduces the research content and illustrates the innovations of this paper. Section 2 introduces the research significance and related background of the 4-UPUS parallel mechanism ankle rehabilitation training robot, and extends the purpose of this research. Section 3 introduces the overall design of the ankle joint rehabilitation robot and the working principle of key components. Section 4 carries out theoretical calculation and analysis on the key technologies in this design, and carries out static analysis and modal analysis on the load-bearing part of the device. The working space and motion performance of the parallel mechanism are discussed. Taking the UPUS single branch chain as the analysis object, the kinematic image of the corresponding surface in the working space of the mechanism is solved, which verifies the correctness of the kinematics theory solution and the feasibility of simulation verification. Section 5 is the conclusion part.

\section{Related work}

The ankle joint is one of the important joints of the human body. The survey shows that ankle joint injuries account for $7 \%$ to $10 \%$ of the total number of patients admitted to the emergency department every day [1-4]. Ankle fracture patients are more common clinically, and the incidence accounts for $4 \%$ to $5 \%$ of systemic fracture injuries [5-6].For the elderly, as the age increases, the flexibility of the limbs gradually decreases, and severe patients may have foot circulation disorders[7-9].Ankle sprains are also a common sports injury. It is difficult to conduct timely and standardized rehabilitation after ankle joint injury, and it is difficult to guarantee the duration of rehabilitation training. When running or jumping, the ankle joint bears huge load and impact, which is a fragile part of the human body [10-12].The causes of illness are mostly caused by indirect violent causes such as eversion, external rotation or inversion. If the treatment method is not selected properly, it will seriously reduce the patient's quality of life [13-14]. At present, in rehabilitation medicine, functional training of joint dysfunction and spasticity requires artificial treatment of ankle joint training[15-16].They are based on the theory of continuous passive motion, stimulate the human body's natural resilience, play a role in tissue compensation, and restore the original functions of joints to the greatest extent [17]. An- 
kle rehabilitation aids have the advantages of labor-saving, high accuracy, and fast recovery. The recovery time is three or four times shorter than manual activity therapy [18-19].With the ankle joint rehabilitation machine, it can be self-adjusted according to the degree of ankle joint dysfunction and the required range of motion. It will not increase the patient's pain and re-injury of the ankle joint, and is of great significance to the early rehabilitation of the ankle joint.

Therefore, in order to meet the application requirements of ankle joint rehabilitation equipment, an ankle joint rehabilitation robot with excellent performance has been developed. It not only allows patients to receive standardized and adequate rehabilitation training, but also alleviates the country's problem of "more patients and fewer rehabilitation doctors". A series of ankle rehabilitation equipment has been developed on the market.

Kumar introduced a new type of $2 S P R R+1 U$ parallel mechanism. The branch chain composed of the spherical pair, the rotating joint and the universal hinge has a certain working space and flexibility, and its kinematics analysis has been carried out [20].Shahid discussed robotic equipment for treadmill and ground training and platform-based parallel ankle robots [21]. Liu proposed a four-degree-of-freedom generalized spherical parallel mechanism, and analyzed whether the kinematics of the mechanism meets the needs of ankle joint rehabilitation [22].Zhi proposed a 3-PSP parallel mechanism, and established a mathematical model of the parallel part of the robot based on the screw theory. Then get the inverse kinematics, and analyze the reciprocal distortion, Jacobian matrix and the singularity of the robot [23].Wang C. studied a kind of transmission branch chain of SPS mechanism. The robot uses the center ball pin pair as the main support to simulate the movement of the ankle joint. The upper platform and the center ball pin pair constitute a mirror image of the patient's ankle joint. It has two degrees of freedom (DOF) connected to the lower platform, which can realize three kinds of movement, and the dorsiflexion, plantarflexion movement and varus and valgus movement are separated, which conforms to the physiological characteristics of the human body [24].Wang D., Choi, JAzar, etc. have respectively developed and verified a new type of foot and ankle bionic dynamics simulator. It is a movable 6-DOF parallel mechanism, URR parallel mechanism and 2R1T branch chain [25-28].Dong M. developed a 2-UPS parallel mechanism whose movement in two directions is driven by two linear motors. It can perform rehabilitation training in dorsiflexion/plantarflexion and varus/valgus directions, calibrate the control accuracy of the kinematic model and derive the inverse solution of the position [29].Wang H. proposed a four-degree-of-freedom lower limb rehabilitation robot, which analyzed the human-machine hybrid kinematics model according to the Denavit-Hartenberg (D-H) method, and explored the user-based variable human-machine workspace. The trajectory tracking experiment of the prototype verifies the trajectory planning method and the human body joint analysis method [30].Based on the theory of generalized function set, Zhang J. reveals the relationship between the motion characteristics of a rigid body and a point on the rigid body. It is determined that the robot body can realize the motion characteristics of the six-dimensional ankle [31]. Kumar S, Li Y, Zhao C proposed a motion mechanism with three degrees of freedom, which has the advantages of high rigidity, simple and strong structure, and good stress distribution [32-34].Zhou C developed a mechanism based on parallel kinematics. By comparing the kinematics analysis of the series mechanism, the performance evaluation of the Jacobian matrix of the speed relationship between the ankle joint and the motor was carried out on the two mechanisms [35]. Yu, Li Y, Lu B, Chen $\mathrm{Z}$, and Cao $\mathrm{Y}$ respectively carried out kinematics analysis on the mechanism to solve the problems of total inverse, rotational inverse and forward kinematics [36-40].

The disadvantages of the ankle joint rehabilitation robot currently under study are:

For general parallel ankle joint rehabilitation robots, the decoupling of kinematics and dynamics is poor, and trajectory control is difficult, which affects the application and promotion of the machine and the effect of rehabilitation training. Most ankle rehabilitation robots have a small range of motion, and the degree of freedom of motion can only 
be used for dorsiflexion/plantar flexion rehabilitation training.It can only be used for the two functions of dorsiflexion/plantar flexion, varus/valgus, internal rotation/external rotation, and cannot meet the training postures required by different patients and the comprehensive rehabilitation needs of ankle rehabilitation. The parallel mechanism robot does not match the motion axis of the human ankle joint. The rotation center of many ankle robots is inconsistent with the rotation center of the human ankle joint, and other parts of the lower limbs will move together during training. This is not suitable for practical ankle rehabilitation training and may cause secondary injuries.

In summary, the ankle joint rehabilitation robot is widely used in medical rehabilitation, and can help users achieve the purpose of treatment. The auxiliary exercise can exercise the ankle joint to a certain extent. Its application enhances the user's lower limb strength and the range of motion of the ankle joint, increases the user's coordination ability, solves the current shortage of medical resources, and makes up for the problem of a large number of patients and a small variety of rehabilitation equipment.

\section{Overall Design of Ankle Joint Rehabilitation Robot}

The ankle is the smallest joint at the end of the three main joints of the lower limbs. Although the ankle is directly connected to the foot, the surrounding muscles are not as strong as the hip and knee joints. It is precisely because of the common use of the ankle in human body training and the relative weakness of the surrounding muscles that active rehabilitation training is required after the joint swelling subsides and the pain is relieved. The ankle joint rehabilitation robot is suitable for abnormal ankle joint function caused by various reasons. It can improve joint stiffness and prevent muscle atrophy and muscle adhesion and ligament adhesion of related leg muscle groups.

The various angles of the ankle joint movement are shown in Figure 3.1.The range of motion angle is shown in Table 1.

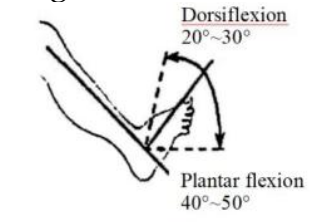

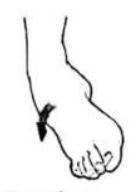

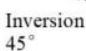

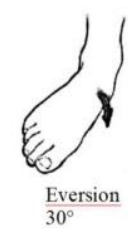
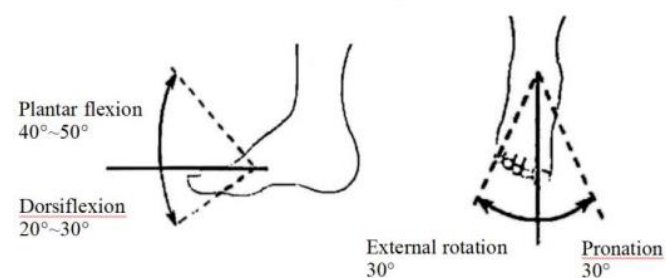

Figure 3.1 Overall schematic diagram of multi-degree-of-freedom ankle rehabilitation training device

Table 1 Range of motion of ankle joint

\begin{tabular}{ccc}
\hline Sports form & Joint & Direction \\
\hline Inversion & $0^{\circ} \sim 45^{\circ}$ & - \\
Eversion & $0^{\circ} \sim 30^{\circ}$ & + \\
Dorsiflexion & $0^{\circ} \sim 30^{\circ}$ & - \\
Plantar flexion & $0^{\circ} \sim 50^{\circ}$ & + \\
External rotation & $0^{\circ} \sim 30^{\circ}$ & + \\
Pronation & $0^{\circ} \sim 30^{\circ}$ & - \\
\hline
\end{tabular}

The working principle of the 4-UPUS parallel ankle joint rehabilitation training robot is that the human body sits on a chair, raises both legs and places it on the ankle joint rehabilitation training robot. After the feet are put on, the ankle joint rehabilitation training can be carried out. It consists of three modules, namely 4-UPUS parallel mechanism, six-link module and foot fixing device. 
The independent design of the two feet realizes the recovery of the left and right ankles separately, and at the same time increases the leg fixation device, which is more practical. The treatment methods can be adjusted according to the different degree of damage of the patient, and the best rehabilitation plan can be formulated.

The use of multiple links in the six-link mechanism module can effectively overcome the lack of internal and external rotation of the ankle joint in the traditional model. Its rotating pairs can be independent of each other, have completely isotropic kinematics, and the control principle is simple.

The overall structure diagram is shown in Figure 3.2.

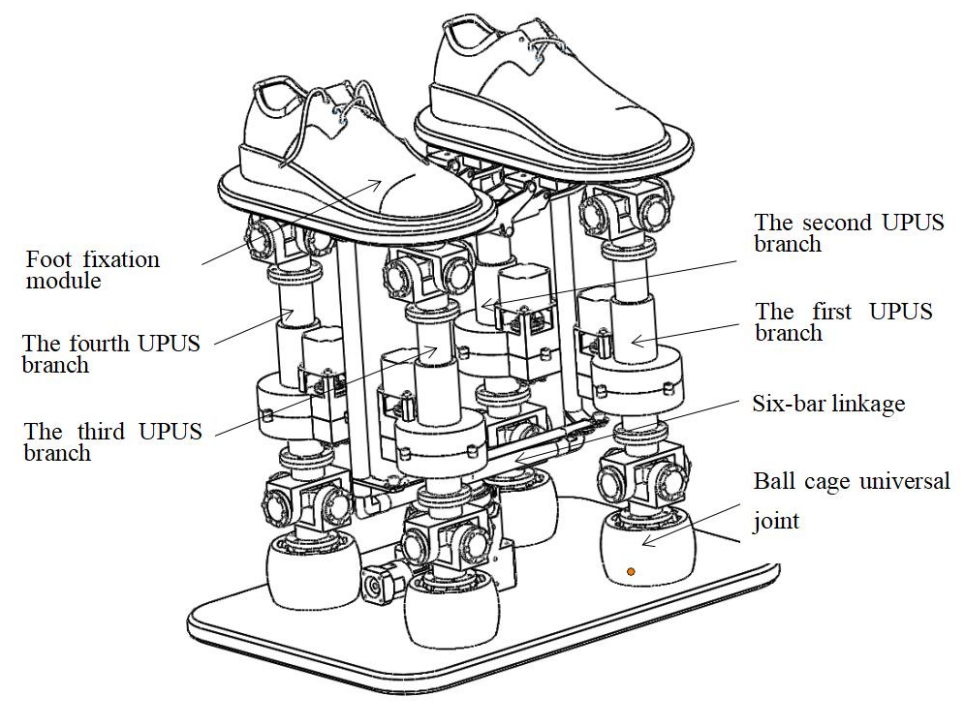

Figure 3.2 Overall schematic diagram of 4-UPUS parallel ankle joint rehabilitation training robot

\subsection{UPUS branch chain structure design}

The UPUS branch chain module mainly includes cross shaft universal coupling, first reducer, servo motor, ball cage universal joint, torque sensor, and displacement sensor. The structure of UPUS branch chain is shown in Figure 3.3.

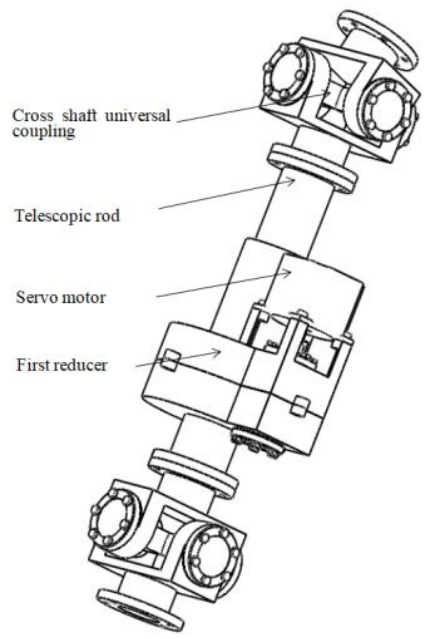

Figure 3.3 UPUS branch chain

The UPUS branch chain is a part that realizes variable-angle power transmission, and is used for the position where the direction of the transmission axis needs to be changed. It is an important part of the universal transmission device of the entire reha- 
bilitation training system. It is fastened to the foot fixing module by a hexagonal screw and plays the role of ankle dorsiflexion and plantar flexion. It has a certain lifting length to ensure that the ankle joint movement components have enough coupling space, which conforms to the physiological characteristics of the human ankle joint movement. One end of the upper cross shaft universal coupling is fastened to the foot fixing module, and one end is fastened to the lifting rod. The output driving force of the servo motor performs the function of raising and lowering the lifting rod through the first reducer. Since the angle and strength of the ankle joint dorsiflexion and plantar flexion are different when the human body performs gait movement, different fuzzy PID control is adopted. One end of the lower cross-shaft universal joint is connected with the ball-cage universal joint, so that the entire universal joint lifting rod has sufficient paranoid mobility, and the movable components will not be restricted when the ankle joint is rotated internally or externally .

\subsection{Structural design of six-bar linkage module}

The six-link mechanism module mainly includes a permanent magnet DC motor, a second reducer, a rotating shaft, a driving rod, a multi-link mechanism, a transmission shaft, and upper and lower movable hinges. The modular structure design of the six-bar linkage mechanism is shown in Figure 3.4.

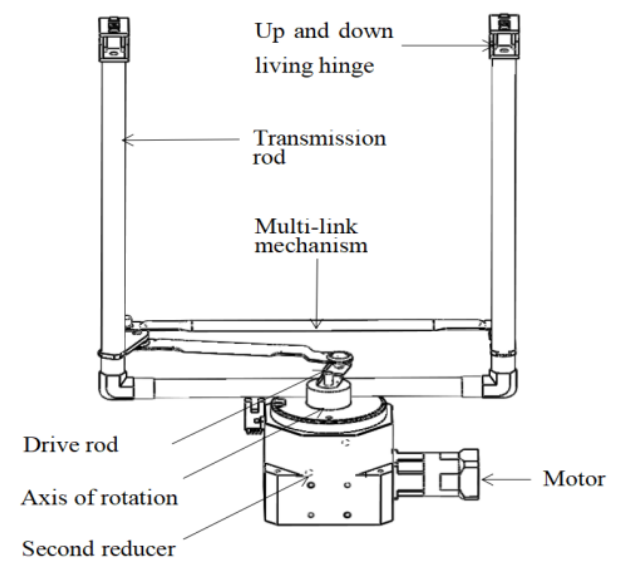

Figure 3.4 Schematic diagram of the six-bar linkage module structure

The six-bar linkage module is mainly linkage transmission, which is responsible for the internal and external rotation of the ankle joint for rehabilitation. The permanent magnet type DC motor is fixed on the support base, and the torque generated by the permanent magnet type DC motor is output to the second reducer after deceleration, and the rotating shaft is driven to rotate to form a high pair. The lower horizontal bottom rod of the multi-link mechanism is used as the frame, and its center position is fixed with the rotating shaft. The two ends of the horizontal bottom rod are connected with one end of the two short rods to form a movable rotating lower pair. The rotating shaft is connected and driven by key positioning. The lower end of the left short rod is connected with the driving rod, and the upper end of the left short rod is connected with the long connecting rod to form a multi-link mechanism.

The rotation of the rotating shaft drives the drive rod to move, and the continuous rotating motion is changed to left and right swinging motion through a multi-link mechanism. The two short rods are connected with the vertical transmission rod for the function of transmitting torque, and the vertical transmission rod is connected with the upper and lower movable hinges. The lower-upper hinge can prevent interference during dorsal extension and plantar flexion of the ankle joint. At the same time, it can transmit the torque in the horizontal plane. The angle sensor is installed in it, which can effectively collect the rotation angle of the ankle joint during internal and external rotation. It can monitor the range of ankle joint rotation in real time, and adjust and set the 
best rehabilitation program accordingly. Complete the internal and external rotation of the ankle joint according to a certain exercise cycle.

\subsection{Foot fixing device}

The foot fixing module includes a foot pedal, a shoe wearable device, and an elastic hinge. The elastic hinge is a rubber hinge made of non-metallic materials, which has a certain degree of tensile ductility and rigidity.

The foot fixing device is mainly an ankle joint rehabilitation movement receiving mechanism. The shoe-type wearing device is fixed on the foot pedal, so that the patient's feet can be worn comfortably, and its size can meet the needs of men and women with various foot sizes to wear shoes. The hinge is fixed under the foot pedal, and a deep groove ball bearing is assembled therein so that a rotating pair can be formed in the axial direction and has a fastening force in the radial direction. The hinge opening angle can reach 90 degrees, which can complete the opening and closing actions well. When the left foot is raised, and the right foot is not moving, the hinge pulling action can realize the foot inversion and eversion function. The hinge is assembled with a spring. When the multi-link actuator completes the rehabilitation training of internal rotation and external rotation of the ankle joint, the compression and extension of the spring can make the entire system device not restrained. The structure of the foot fixing device is shown in Figure 3.5.

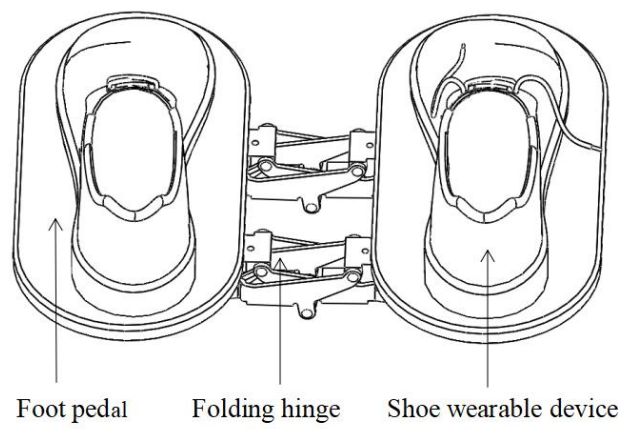

Figure 3.5 Schematic diagram of the foot fixing device

\subsection{Exercise mechanism and rehabilitation program}

As shown in Figure 3.6, the left foot mechanism is defined as the first lifting rod near the toe and the second lifting rod near the heel. When the first lifting rod is raised to a certain height, the second lifting rod remains stationary. With the raising of the first lifting rod, the two shafts of the universal joint are not on the same axis and offset, and the universal joint of the stationary second lifting rod maintains the same included angle to form the height difference of the foot around the ankle joint, And the maximum included angle with the horizontal plane range can be $20^{\circ} \sim 30^{\circ}$, so as to drive the upper foot pedal and shoe wear device to form the dorsiflexion action of the back of the foot close to the front of the calf, and the dorsiflexion training of the ankle joint of the foot can be completed.

When the first lifting rod is in a static state and the second lifting rod keeps rising at this time, as the second lifting rod is raised, the two shafts of the universal coupling are not on the same axis and deviate. It maintains the same included angle with the universal coupling of the stationary second lifting rod to form the height difference of the foot around the ankle joint, and the maximum included angle with the horizontal plane can be $40^{\circ}-50^{\circ}$. Therefore, the upper foot pedal 301 and the shoe wearable device 302 are driven to form a plantar flexion movement away from the front of the calf, and the plantar flexion movement of the ankle joint of the foot can be completed. The left foot mechanism and the right foot mechanism work at the same time to complete the dorsiflexion and plantarflexion training of the left and right ankle joints. 
The movable hinge is connected with the middle end of the bottom of the foot pedal. When the left foot mechanism and the right foot mechanism work at the same time to complete the left and right ankle joint dorsiflexion and plantar flexion rehabilitation training, the hinge rotates with it, so that the movement does not occur during the dorsiflexion and plantar flexion rehabilitation training. Interference.

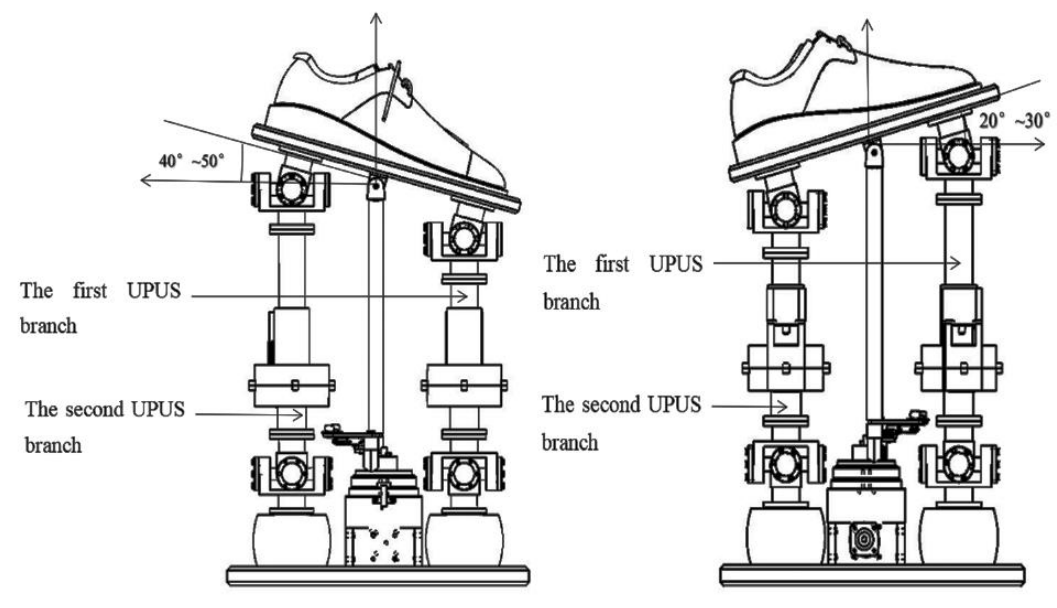

Figure 3.6 Schematic diagram of left and right ankle joint dorsiflexion and plantar flexion movement

As shown in Figure 3.7, the right foot mechanism is defined as the third lifting rod near the toe and the fourth lifting rod near the heel. When the first and second universal lifting rods of the left foot mechanism are raised at the same time, the third and fourth universal lifting rods of the right foot mechanism are in a static state, and the left foot mechanism drives the right foot mechanism through two hinges to cause an angular deviation. When the left foot mechanism moves to a certain height, the right foot mechanism is correspondingly driven to a certain angle. At this time, the inner edge is raised, the outer edge is lowered, and the sole of the right foot is inward and the back of the instep is outward. And the maximum critical angle with the horizontal plane range can be $45^{\circ}$. When the third and fourth universal lifting rods of the right foot mechanism are raised at the same time, the first and second universal lifting rods of the left foot mechanism are in a static state at this time. The right foot mechanism drives the left foot mechanism to complete the action through two hinges. When the right foot mechanism moves to a certain height, the left foot mechanism is correspondingly driven to a certain angle. At this time, the inner edge is raised, the outer edge is lowered, and the sole of the left foot is inward and the back of the instep is outward. The maximum critical angle with the horizontal plane range can be $45^{\circ}$. Can complete the inversion training of the ankle joint of the foot.

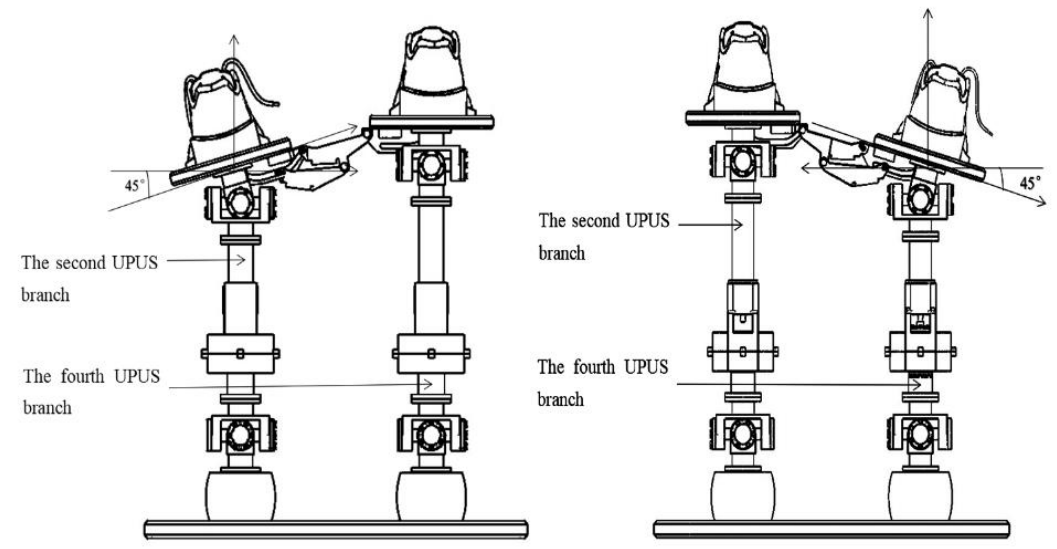


Figure 3.7 Schematic diagram of the varus movement of the left and right ankle joints

As shown in Figure 3.8, when the first and second universal lifting rods of the left foot mechanism descend at the same time, the third and fourth universal lifting rods of the right foot mechanism are in a static state at this time, and the left foot mechanism is driven by two hinges. The right foot mechanism has an angular deviation. When the left foot mechanism drops to a certain height, the right foot mechanism is correspondingly driven to a certain angle. The outer edge of the foot is raised and the inner edge is lowered, with the sole of the right foot facing inward and the back of the instep facing outward. The maximum critical angle with the horizontal plane range can be $15^{\circ}$. When the third and fourth universal lifting rods of the right foot mechanism descend at the same time, the first and second universal lifting rods of the left foot mechanism are in a static state, and the right foot mechanism drives the right foot mechanism through two hinges to complete the action. When the right foot mechanism drops to a certain height, the left foot mechanism is correspondingly driven to a certain angle, the outer edge of the foot is raised, the inner edge is lowered, and the sole of the left foot is inward and the back of the instep is outward. The maximum critical angle with the horizontal plane can be $15^{\circ}$, and the valgus training of the ankle joint of the foot can be completed.

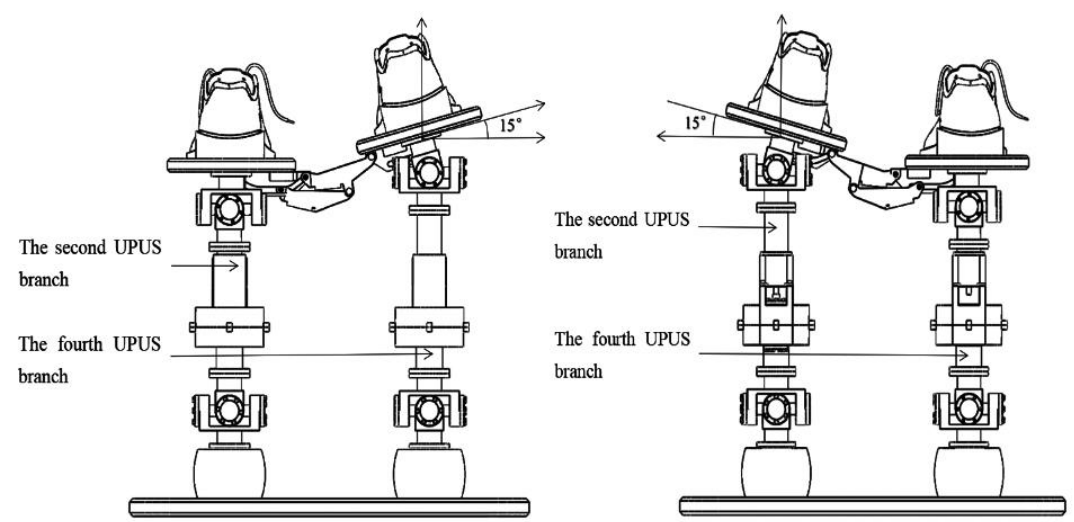

Figure 3.8 Schematic diagram of valgus movement of left and right ankle joints

Refer to Figure 4.5. When the rotating shaft of the six-bar linkage mechanism rotates, it drives the drive rod $\mathrm{AB}$ to make a circular motion, drives the drive rod $\mathrm{BC}$ to push $\mathrm{DE}$ to swing, and FG also swings under the action of the connecting rod. The whole mechanism forms a reciprocating movement that swings from side to side. The rehabilitation actions of internal and external rotation of the ankle joint can be completed. The schematic diagram of internal rotation and external rotation is shown in Figure 3.9.

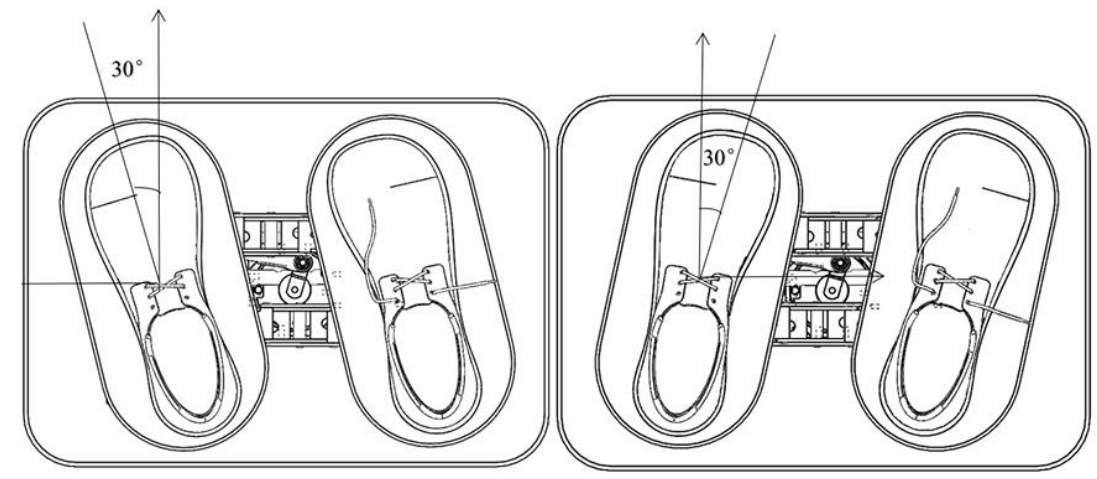

Figure 3.9 Schematic diagram of internal rotation and external rotation of the left and right ankle joints

\section{Key technology research and discussion}

\subsection{Finite Element Static Analysis}


Considering that the patient's legs need to be placed on the robot system, the overall structure is subjected to static stress analysis to verify whether it has a certain load-bearing capacity to meet the requirements of the working conditions. This article takes the overall structure of the robot system as the analysis object, and most of the loads are from the weight of the patient's legs. The weight of the leg of the trainer on the overall structure is regarded as a uniform load. In this design, the weight bearing range of the human leg is $0 \sim 30 \mathrm{~kg}, \mathrm{~g}=9.8 \mathrm{~m} / \mathrm{s}^{2}$, and the left and right bearing mechanisms each bear the weight of one leg, so the load is set to $150 \mathrm{~N}$.

For the elastic deformation of the running frame, the relationship between force and displacement satisfies the following formula:

$$
[K] \times\{\delta\}=\{F\}
$$

Among them: $\{F\}$ is the total load array; $[K]$ is the system structure stiffness matrix; $\{\delta\}$ is the system node displacement array;

The established finite element model analysis of the overall structure is shown in Figure 4.1 and Figure 4.2.

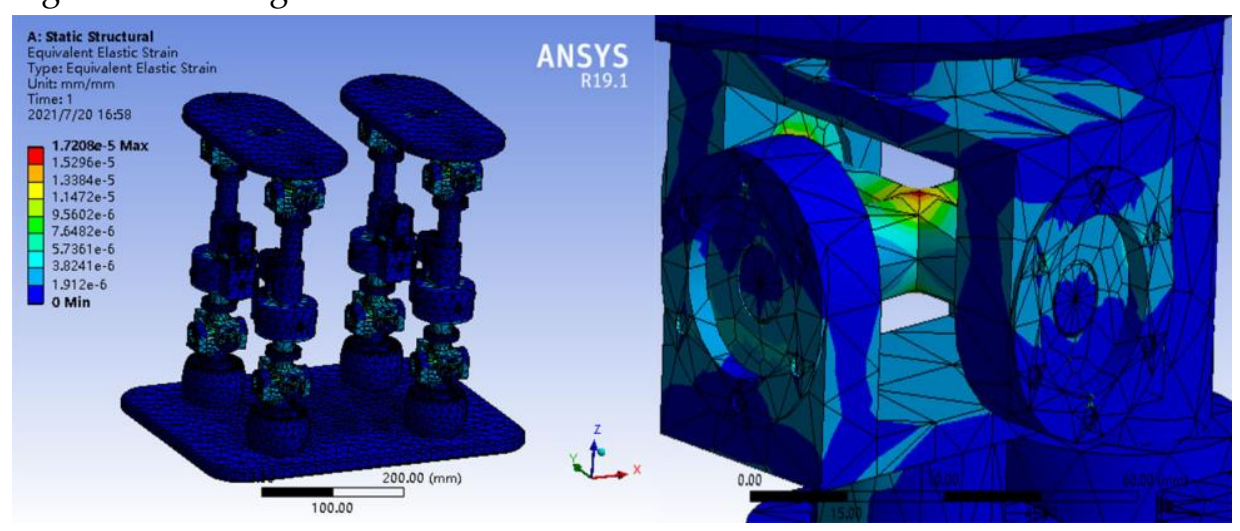

Figure 4.1 Equivalent stress diagram

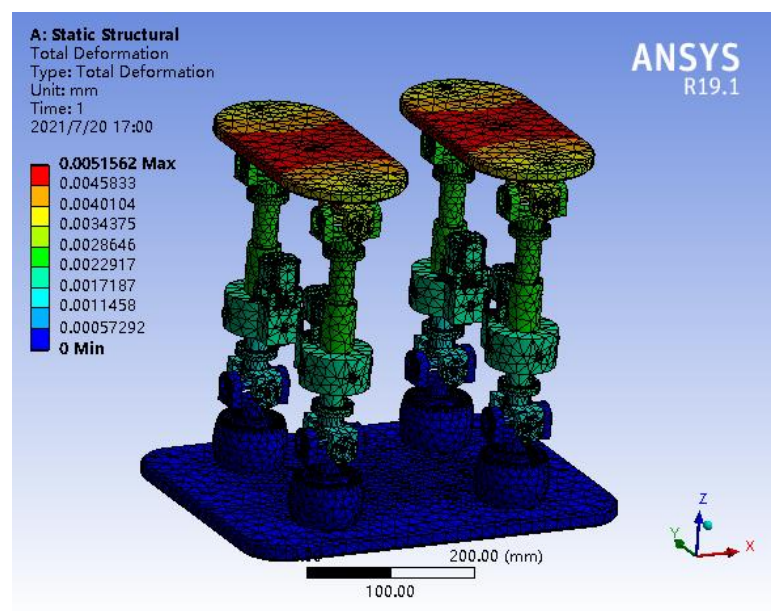

Figure 4.2 Schematic diagram of total deformation

The static analysis results show that from the equivalent stress diagram, it can be seen that under the condition of applying a simulated load, the calculation and operation results of the stress distribution of the overall structure show that the overall stress is small and uniformly distributed. It is mainly concentrated at the center of the cross axis of the universal joint. Although the stress here is slightly larger, the dynamic yield stress of the overall structure is much smaller than the yield strength limit of the material. The maximum strength of the frame in this state is $3.4407 \mathrm{Mpa}$, and the maximum defor- 
mation is $0.0051562 \mathrm{~mm}$. The dynamic load stress intensity at all parts of the frame is much smaller than the yield limit of 235Mpa, and the deformation caused by the applied load can be ignored. It can be seen from the total deformation diagram that the main deformation is at the center of the feet in the foot fixing device, where the overall structure is weak. As an optimized design, in the overall design, the center of the foot fixing device has the transmission rod of the crank and rocker swing module as a support, which can well overcome the deformation caused by the insufficient yield strength of the material. Therefore, the mechanical performance requirements can be well met in the fully loaded state or in the incompletely loaded static state.

\subsection{Motion analysis of six-bar linkage structure}

\subsubsection{Motion analysis of six-bar linkage}

In the robot system, the internal rotation and external rotation of the ankle joint rehabilitation are mainly performed by a multi-link motion mechanism. As shown in Figure 4.3 , the motion link mechanism mainly performs the reciprocating motion of left and right swing relative to the external rotation and internal rotation.

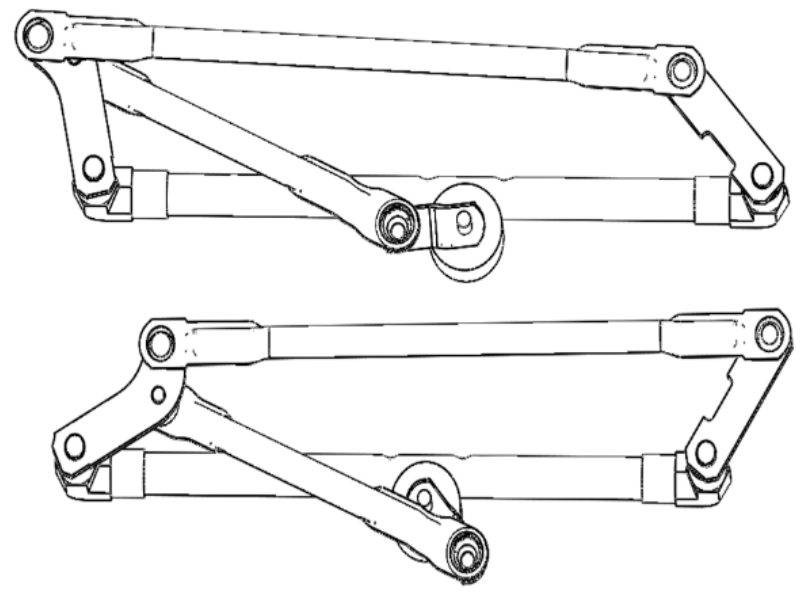

Figure 4.3 Schematic diagram of six-bar linkage mechanism swing

The model is simplified into a linkage mechanism, and the solution is solved according to the different assumptions taken.

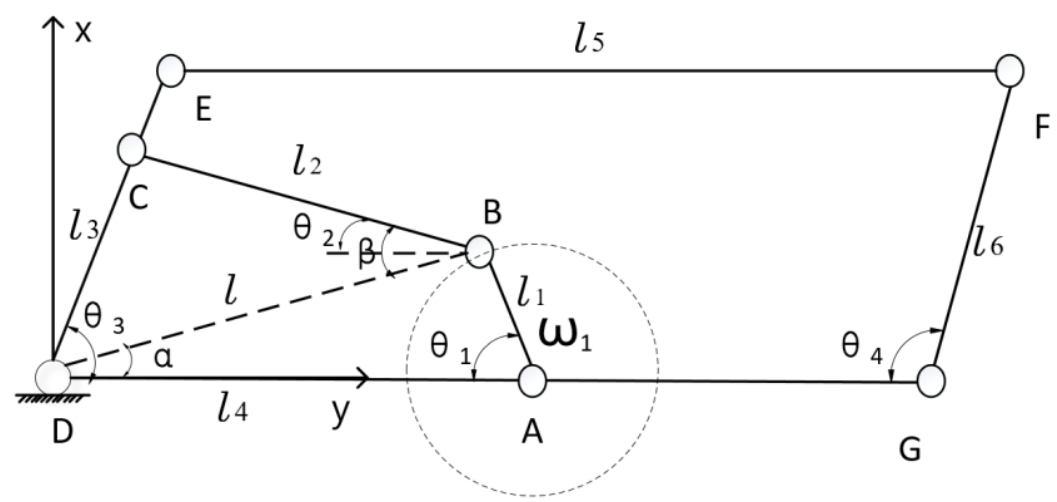

Figure 4.4 Simplified model of the model

The relative position of each rod in space can be roughly determined by the schematic diagram of the mechanism. As shown in Figure 4.3, roughly determine the relative position of each rod in space. Among them, point $A$ is the input hinge fulcrum, the $A B$ rod is the input rod, and its angular velocity is $\omega_{1}$, which drives the movement of the $B C$ rod and promotes the movement of the $D E$ rod, so that the $E F$ and $F G$ move to form a reciprocating movement of left and right swings, thereby completing the internal rotation of the ankle joint. Rehabilitation of external rotation.

From geometric relations, the following equations can be established: 


$$
\begin{gathered}
\left\{\begin{array}{l}
l_{4}-l_{1} \cos \theta_{1}=l_{3} \cos \theta_{3}+l_{2} \cos \theta_{2} \\
l_{1} \sin \theta_{1}=l_{3} \sin \theta_{3}+l_{2} \sin \theta_{2}
\end{array}\right. \\
l=\sqrt{l_{3}^{2}+l_{4}^{2}-2 l_{3} l_{4} \cos \theta_{3}} \\
\left\{\begin{array}{l}
\cos \alpha=\frac{\left(l_{4}+l_{3} \cos \theta_{3}\right)}{l} \\
\sin \alpha=\frac{l_{3} \sin \theta_{3}}{l}
\end{array}\right. \\
\left\{\begin{array}{l}
\cos \beta=\frac{\left(l_{2}^{2}+l^{2}-l_{1}^{2}\right.}{2 l_{2} l} \\
\sin \beta=\sqrt{1-\cos ^{2} \beta}
\end{array}\right.
\end{gathered}
$$

After the coordinate system is established, point $A$ is used as the input terminal, and $a, \beta, \theta_{1}, \theta_{2}, \theta_{3}$ are defined in the closed vector $A B C D$ four link, so that the position of each point can be obtained.

$$
\begin{gathered}
\left\{\begin{array} { l } 
{ x _ { C } = l _ { 3 } \operatorname { c o s } \theta _ { 3 } } \\
{ y _ { C } = l _ { 3 } \operatorname { s i n } \theta _ { 3 } }
\end{array} \quad \left\{\begin{array}{l}
x_{A}=l_{4} \\
y_{A}=0
\end{array}\right.\right. \\
\left\{\begin{array}{l}
x_{B}=x_{C}+l_{2} \cos \theta_{2}=l_{3} \cos \theta_{3}+l_{2} \cos (\beta-\alpha) \\
=l_{3} \cos \theta_{3}+l_{2}(\cos \beta \cos \alpha+\sin \beta \sin \alpha) \\
y_{B}=y_{C}+l_{2} \sin \theta_{2}=l_{3} \sin \theta_{3}+l_{2} \sin (\beta-\alpha) \\
=l_{3} \sin \theta_{3}+l_{2}(\sin \beta \cos \alpha-\cos \beta \sin \alpha)
\end{array}\right. \\
\left\{\begin{array}{l}
x_{F}=l_{3} \cos \theta_{3}+\left(l_{6}-l_{3}\right) \cos \theta_{3}+l_{5} \cos \theta_{2} \\
y_{F}=l_{3} \sin \theta_{3}+l_{5} \sin \theta_{2}
\end{array}\right.
\end{gathered}
$$

By formula (4.6) and formula (4.7) to determine the positions of the dynamic positioning points $B$ and $C$, the mechanism can be moved. According to formula (4.8), the movement position of point $F$ can be calculated.

$$
\begin{aligned}
\theta_{2}=\arctan \frac{\sin \theta_{2}}{\cos \theta_{2}} & =\arctan \frac{\sin \beta \cos \alpha-\cos \beta \sin \alpha}{\cos \beta \cos \alpha+\sin \beta \sin \alpha} \\
\theta_{1} & =\arctan \frac{y_{B}-y_{A}}{x_{B}-x_{A}}
\end{aligned}
$$

4.2.2 Speed analysis

Derivation from equation (4.3) to time $t$, we get 


$$
\left\{\begin{array}{l}
l_{1} \dot{\theta}_{1} \cos \theta_{1}=-l_{3} \dot{\theta}_{3} \sin \theta_{3}-l_{2} \dot{\theta}_{2} \sin \theta_{2} \\
-l_{1} \dot{\theta}_{1} \cos \theta_{1}=l_{3} \dot{\theta}_{3} \cos \theta_{3}+l_{2} \dot{\theta}_{2} \cos \theta_{2}
\end{array}\right.
$$

$\dot{\theta}_{1}=\omega_{1}, \quad \dot{\theta}_{2}=\omega_{2}, \quad \dot{\theta}_{3}=\omega_{3}$ are the angular velocities of rods $A B, B C$, and $C D$ respectively.

$$
\begin{gathered}
\omega_{2}=\frac{-l_{3} \sin \left(\theta_{1}+\theta_{3}\right)}{l_{2} \sin \left(\theta_{1}-\theta_{2}\right)} \omega_{1} \\
\omega_{3}=\frac{l_{1} \sin \left(\theta_{1}+\theta_{2}\right)}{l_{3} \sin \left(\theta_{3}-\theta_{2}\right)} \omega_{1}
\end{gathered}
$$

Derive the time $t$ from equation (4.8), and calculate the speed at point $F$

$$
\begin{gathered}
v_{\mathrm{F} x}=-l_{3} \omega_{3} \sin \theta_{3}-l_{5} \omega_{2} \cos \theta_{2} \\
v_{\mathrm{Fy}}=l_{3} \omega_{3} \cos \theta_{3}+l_{5} \omega_{2} \cos \theta_{2} \\
v_{F}=\sqrt{v_{F x}^{2}+v_{F y}^{2}}
\end{gathered}
$$

According to the same steps, the second derivative of the time $t$ can be obtained from the equation system, and the angular acceleration of the rod $B C$ and $C D$ can be obtained. $\ddot{\phi}_{1}=0$, we can get

$$
\begin{aligned}
& \varepsilon_{2}=\frac{l_{3} \omega_{3}^{2} \cos \left(\theta_{1}+\theta_{3}\right)-l_{2} \omega_{2}^{2} \cos \left(\theta_{1}+\theta_{2}\right)-l_{1} \omega_{1}^{2}}{l_{2} \sin \left(\theta_{1}+\theta_{2}\right)} \\
& \varepsilon_{3}=\frac{l_{1} \omega_{1}^{2} \cos \left(\theta_{1}-\theta_{2}\right)+l_{2} \omega_{2}^{2}-l_{3} \omega_{3}^{2} \cos \left(\theta_{3}-\theta_{2}\right)}{l_{3} \sin \left(\theta_{3}-\theta_{2}\right)}
\end{aligned}
$$

Calculating the second derivative of equation (4.7), the acceleration can be obtained as

$$
\begin{gathered}
a_{F x}=-l_{3} \omega_{3}^{2} \cos \theta_{3}-l_{5} \omega_{2}^{2} \cos \theta_{2}-l_{5} \varepsilon_{2} \sin \theta_{2} \\
a_{F y}=-l_{3} \omega_{3}^{2} \sin \theta_{3}-l_{5} \omega_{2}^{2} \sin \theta_{2}+l_{5} \varepsilon_{2} \cos \theta_{2} \\
a_{F}=\sqrt{a_{F x}^{2}+a_{F y}^{2}}
\end{gathered}
$$

4.2.3 Simulation analysis of six-bar linkage 
The ankle joint is the main joint for the human body to complete gait movement during walking. The ankle joint has the characteristics of external rotation and internal rotation during normal walking. The range of internal rotation angle is $0^{\circ} \sim 30^{\circ}$; the range of external rotation angle is $0^{\circ} \sim 50^{\circ}$. When used in healthy people, the angle of motion at the ankle should be controlled within this range. However, considering that the patient's ankle joint has basic motor dysfunction, to avoid unnecessary secondary injury, its angle of motion should not be too large.

Verify that the motion angle of the linkage mechanism conforms to the range of the ankle joint motion angle when the human body moves normally, to explore the control law of the original mover, and verify the rationality of the mechanism design. The kinematics images of the mechanism are shown in Figures 4.5 to 4.10 .

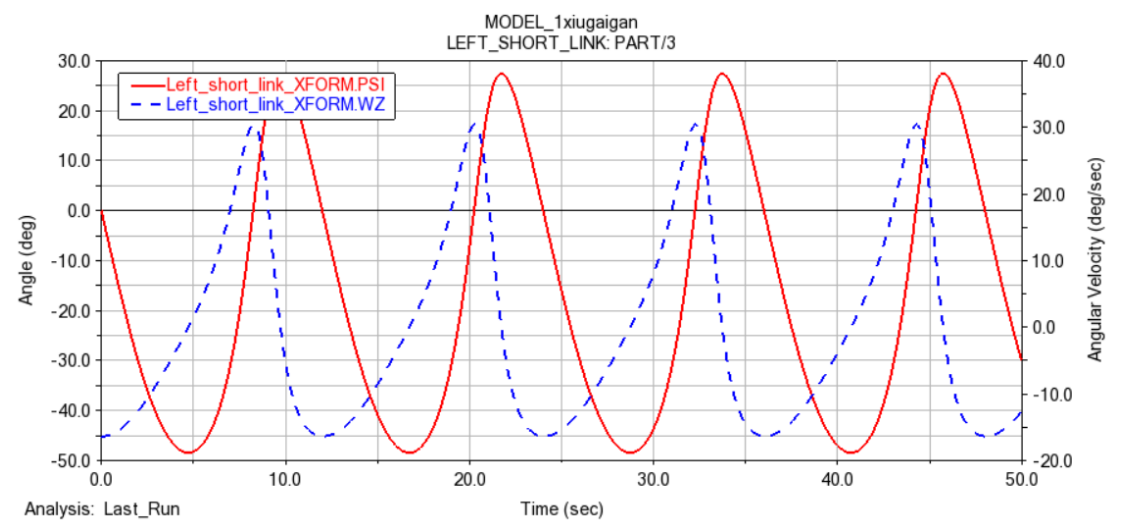

Figure 4.5 $D E$ left link movement angle diagram

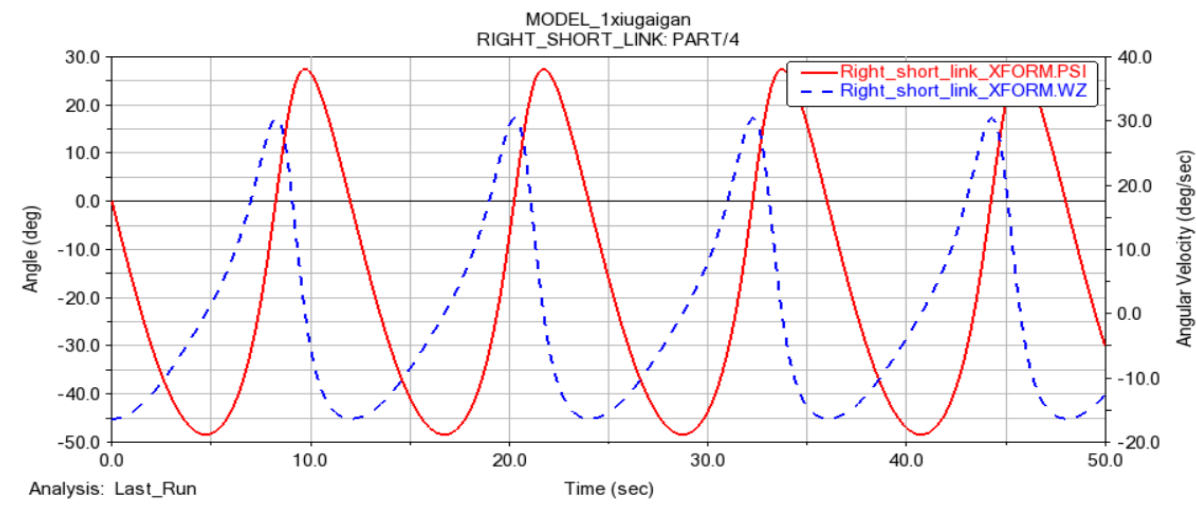

Figure 4.6 FG right link movement angle diagram

The curve in Figure 4.5 reflects the trend of the movement angle of the left link over time. The movement angle corresponds to the movement angle of the ankle joint of the left foot. It can be seen that the angle of internal rotation of the ankle joint is $0^{\circ} \sim+30^{\circ}$, and it reflects that the angle of external rotation is between $-50^{\circ} \sim 0^{\circ}$. The range of angle fluctuation is within the range of motion of the ankle joint in the normal gait of the human body.

The corresponding movement angle in Figure 4.6 corresponds to the movement angle of the right ankle joint. It can be seen that the angle of internal rotation of the ankle joint is $0^{\circ} \sim+30^{\circ}$, and it reflects that the angle of external rotation is between $-50^{\circ} \sim 0^{\circ}$. It can well simulate the internal rotation and external rotation characteristics of the human ankle joint in gait motion. 


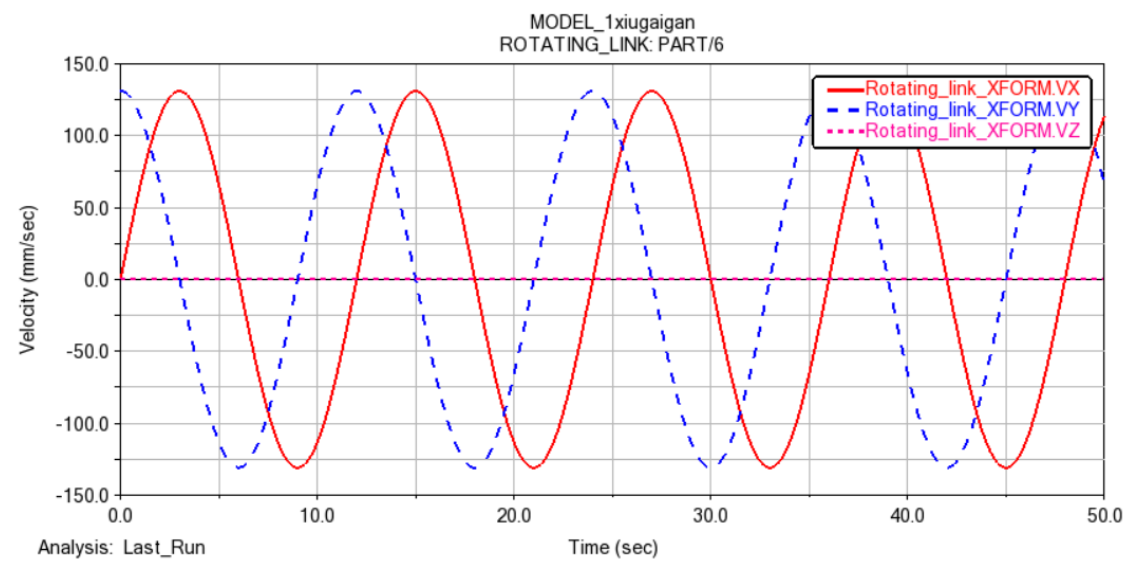

Figure 4.7 Speed image of $A B$ rotating rod

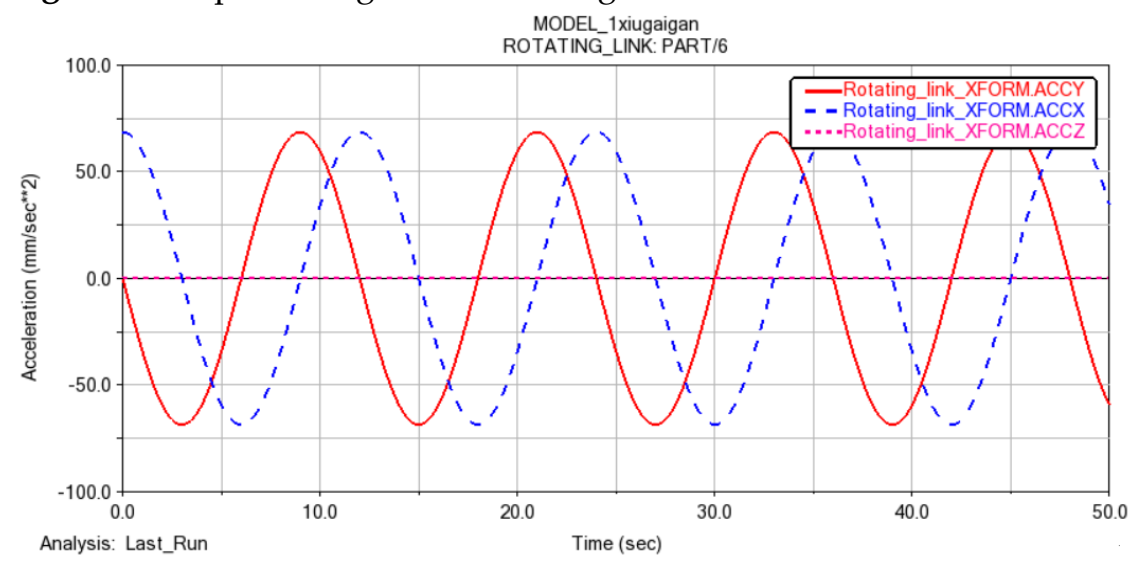

Figure 4.8 Acceleration motion image of $A B$ rotating rod

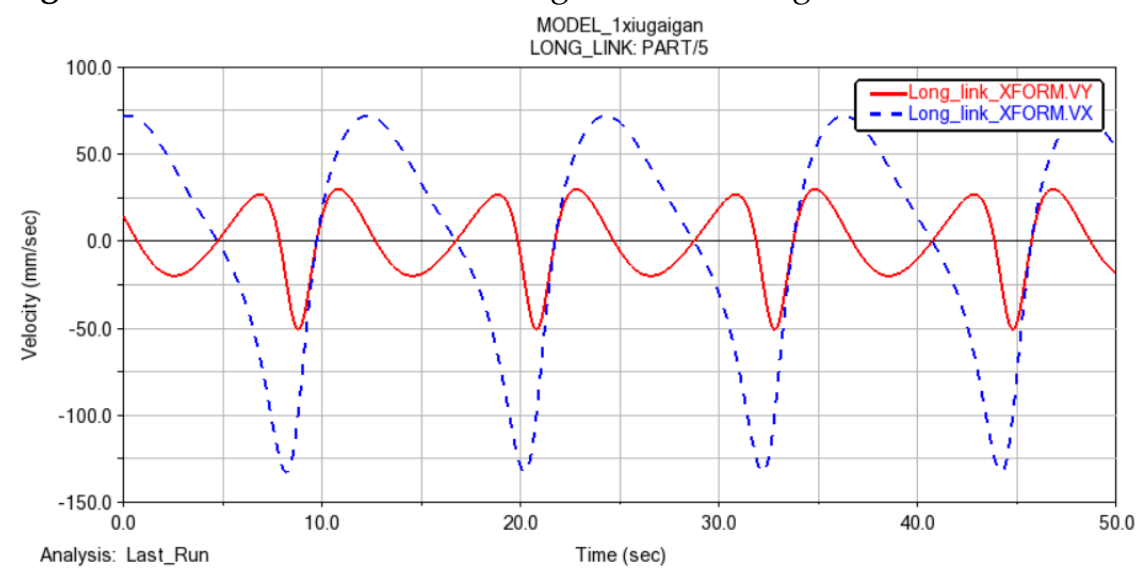

Figure 4.9 Speed diagram of EF long link

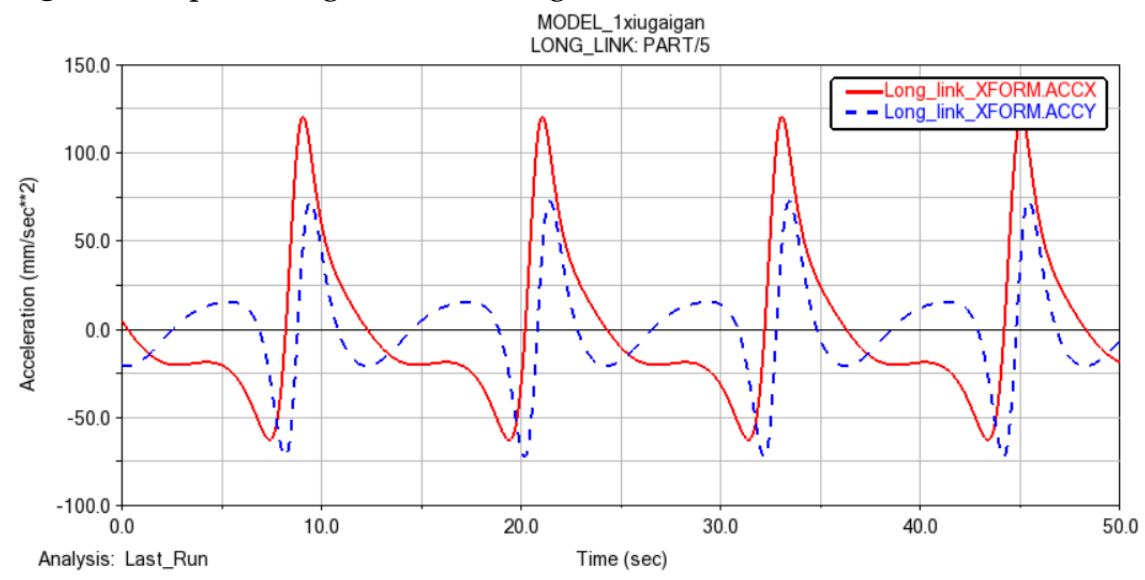


Figure 4.10 Acceleration diagram of $E F$ long link

Studies have shown that the normal walking speed of humans is about $1 \mathrm{~m} / \mathrm{s}$. Considering that the patient's ankle joint suffers from basic motor dysfunction, to avoid unnecessary secondary injury, the value here is smaller than normal and is used for research.

The speed and acceleration of the $A B$ drive rod shown in Figures 4.7 and 4.8 change with time. The curves show the changes in the speed and acceleration of the $A B$ link in the horizontal and vertical directions. From the speed curve, we can see that the maximum speed of the $A B$ drive rod is within $0.1 \mathrm{~m} / \mathrm{s}$.

The speed of the $E F$ long link shown in Figures 4.9 and 4.10 varies with time. The curves show the changes in the speed and acceleration of the EF long link in the horizontal and vertical directions. From the speed curve, we can see that the maximum speed of the $E F$ long link is also within $0.1 \mathrm{~m} / \mathrm{s}$.

It can be concluded that the speed of the $A B$ drive rod and the $E F$ long link can be controlled within the speed range of $1 \mathrm{~m} / \mathrm{s}$, that is, the simulation results are in line with expectations. Therefore, the multi-link structure design is more reasonable, which can satisfy the ankle joint activity during normal gait movement of the human body.

\subsection{Dynamic analysis based on 4-UPUS parallel mechanism}

\subsubsection{Kinematics analysis of parallel mechanism model}

As shown in Figure 4.11, the structure of the static and dynamic platform of the parallel mechanism is a rectangle, the dynamic coordinate system $o-x y z$ is connected to the dynamic platform, and the static coordinate system $0-X Y Z$ is in a fixed state. Let $[x, y, z, \alpha, \beta, \gamma]$ be the displacement and rotation angle of the moving platform coordinate system $\mathrm{M}$ relative to the stationary platform coordinate system $B$.Among them, $[x, y, z]$ is the displacement along the $X, Y$, and $Z$ coordinate axes, and $[\alpha, \beta, \gamma]$ is the Euler angle of the dynamic coordinate system $M$ relative to the static coordinate system $B$.

Since the six-bar linkage device completes internal and external rotation actions, the moving platform of the parallel mechanism has rotational freedom around the $X, Y, Z$ axes and freedom of movement along the $Z$ axis. Then the generalized output of the parallel mechanism moving platform is $[\alpha, \beta, \gamma, z]$.

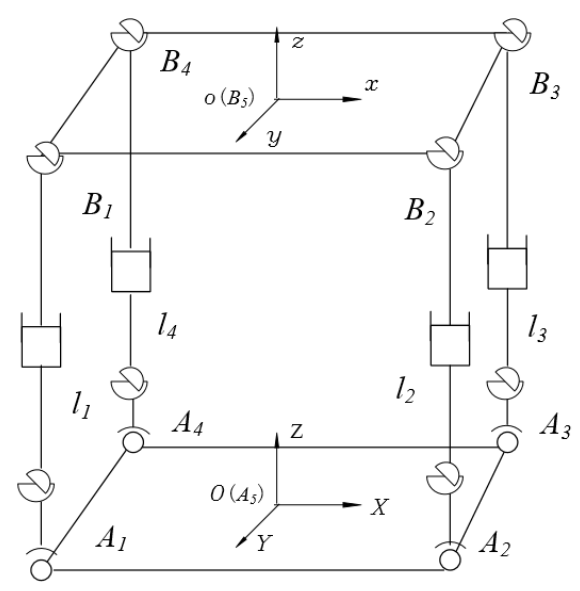

Figure 4.11 4-UPUS parallel mechanism model diagram

The coordinates of $A_{i}$ point in the fixed coordinate system $O-X Y Z$ are:

$$
{ }^{0} A_{i}=\left(A_{i X}, A_{i Y}, A_{i Z}\right)^{T} \quad i=1,2,3,4,5
$$

The coordinates of $\mathrm{Bi}_{\mathrm{i}}$ point in the moving coordinate system $o-x y z$ are: 


$$
{ }^{0} B_{i}=\left(B_{i X}, B_{i Y}, B_{i Z}\right)^{T} \quad i=1,2,3,4,5
$$

The coordinates of point 0 in the fixed coordinate system $0-X Y Z$ are:

$$
p=(x, y, z)^{T}
$$

The coordinates of point $B$ in the fixed coordinate system $0-X Y Z$ are:

$$
{ }^{o} B_{i}=\left(B_{i X}, B_{i Y}, B_{i Z}\right)^{T}={ }^{o} B_{i} R+p-{ }^{o} A_{i}
$$

According to the movement of each branch of the parallel mechanism, the coordinates of the moving platform and the static platform are connected to the origin of the two coordinate systems to form a closed loop. Therefore, the establishment of the geometric vector relationship can analyze the speed and the Jacobian matrix.

The relationship between the input speed $v_{o}$ and the output speed $v_{i n}$ of the 4-UPUS parallel mechanism is

$$
v_{o}=J v_{i n}
$$

$J$ is the Jacobian matrix of the parallel mechanism. When the determinant of $J$ is equal to $0, v_{o}$ cannot be inversely solved by $v_{i n}$, and a singular configuration will appear.

The vertices $b_{i}$ of the moving platform of the 4-UPUS parallel mechanism are expressed as $b_{i}^{m}$ in the dynamic coordinate system and $b_{i}$ in the static coordinate system. The relationship between them is

$$
b_{i}=R_{m}^{B} b_{i}^{m}+o
$$

$R_{m}^{B}$ is the coordinate conversion matrix from the moving coordinate system $M$ to the static coordinate system $B$. Using ZYX Euler angle conversion method, it can be expressed as:

h ${ }_{\mathrm{e}}^{\mathrm{T}} R_{m}^{B}=\left[\begin{array}{ccc}\cos \beta \cos \alpha & \cos \alpha \sin \beta \sin \gamma-\sin \alpha \cos \gamma & \cos \alpha \sin \beta \cos \gamma+\sin \alpha \sin \gamma \\ \cos \beta \sin \alpha & \sin \alpha \sin \beta \sin \gamma+\cos \alpha \cos \gamma & \sin \alpha \sin \beta \cos \gamma-\cos \alpha \sin \gamma \\ -\sin \beta & \cos \beta \sin \gamma & \cos \beta \cos \gamma\end{array}\right]$

e

ctor from the center point o of the moving platform to the vertex $\mathrm{b}$ of the moving platform is

$$
e_{i}=b_{i}-o
$$

Write the velocity of the center point o of the moving platform as 


$$
v=\left[\begin{array}{c}
0 \\
0 \\
\dot{z}
\end{array}\right]=\left[\begin{array}{cccc}
0 & 0 & 0 & 0 \\
0 & 0 & 0 & 0 \\
0 & 0 & 0 & 1
\end{array}\right]\left[\begin{array}{c}
\dot{\alpha} \\
\dot{\beta} \\
\dot{\gamma} \\
\dot{z}
\end{array}\right]=J_{v} v_{0}
$$

In the formula, $G$ represents the generalized speed of the moving platform of the 4-UPUS parallel mechanism.

Using ZYX type Euler angle, the angular velocity $\mathrm{F}$ of the moving platform can be expressed as

$$
\omega=R_{1} \dot{\alpha}+R_{2} \dot{\beta}+R_{3} \dot{\gamma}
$$

$R_{1}, R_{2}, R_{3}$, are the directions of the three rotating shafts when the movable platform is rotating.

$$
\left\{\begin{array}{l}
R_{1}=R_{Z} \\
R_{2}=R(Z, \alpha) R_{Y}=\left[\begin{array}{lll}
-\sin \alpha & \cos \alpha & 0
\end{array}\right]^{T} \\
R_{3}=R(Z \alpha) R(Y \beta) R_{X}=\left[\begin{array}{lll}
\cos \alpha \cos \beta & \sin \alpha \cos \beta & -\sin \beta
\end{array}\right]^{T}
\end{array}\right.
$$

$R_{X}, R_{Y}, R_{Z}$ respectively represent the unit direction vectors of the three coordinate axes of the static coordinate system $B$, so

$$
\omega=\left[\begin{array}{cccc}
0 & -\sin \alpha & \cos \alpha \cos \beta & 0 \\
0 & \cos \alpha & \sin \alpha \cos \beta & 0 \\
1 & 0 & -\sin \beta & 0
\end{array}\right]\left[\begin{array}{c}
\dot{\alpha} \\
\dot{\beta} \\
\dot{\gamma} \\
\dot{z}
\end{array}\right]=J_{\omega} v_{0}
$$

According to the angular velocity $\omega$ of the moving platform and the speed of the center point o of the moving platform, the input speed $v_{i n i}$ of each drive branch can be obtained.

$$
v_{i n i}=v_{i} \delta_{i}=\left(v+\omega \times e_{i}\right) \delta_{i}=\delta_{i} v+\left(e_{i} \times \delta_{i}\right) \omega=J_{r i}\left[\begin{array}{c}
v \\
\omega
\end{array}\right]
$$

\subsubsection{Kinematics simulation verification of parallel mechanism}

For the studied 4-UPUS parallel mechanism, its reachable working space is defined as the set of all moving platform pose parameters $\alpha, \beta, \gamma$ that meet the constraint conditions.

The working space of the mechanism must satisfy a certain symmetry, and the working space of the mechanism can be obtained by combining the inverse kinematics solution of the mechanism. Using the coordinate search method, the points that meet the constraints are determined in the designated area, and then the workspace image is obtained through the coordinates of these points .

When analyzing the working space of the location, the single-open chain analysis method is adopted. Regarding each branch chain as an independent movement, the intersection of the workspace formed by the 4 branch chains is the positional workspace.While satisfying the equation in 4.3.1, the position constraint equation of the parallel mechanism is combined to form the constraint condition of the working space. Record all the points that meet the constraint condition, then the volume composed of these points constitutes the working space of the parallel mechanism.Select a constant 
point in the space and keep it still, let the Euler angles $\alpha, \beta, \gamma$ change, and get all the Euler angles that can reach the fixed position.

Given the attitude angle $\alpha, \beta, \gamma$ find each angle according to $\mathrm{f}(\alpha, \beta, \gamma)$, let

$$
\begin{aligned}
& \alpha_{i}=\alpha_{\min }+i \frac{\alpha_{\max }-\alpha_{\min }}{m-1}(i=0,1, \cdots, m-1) \\
& \beta_{i}=\beta_{\min }+i \frac{\beta_{\max }-\beta_{\min }}{m-1}(i=0,1, \cdots, m-1) \\
& \gamma_{i}=\gamma_{\min }+i \frac{\gamma_{\max }-\gamma_{\min }}{m-1}(i=0,1, \cdots, m-1)
\end{aligned}
$$

Loop $\mathrm{i}$ times, and then search each corner one by one, and finally get the singularity boundary curve of the attitude angle change. As shown in Figure 4.13 and 4.14.

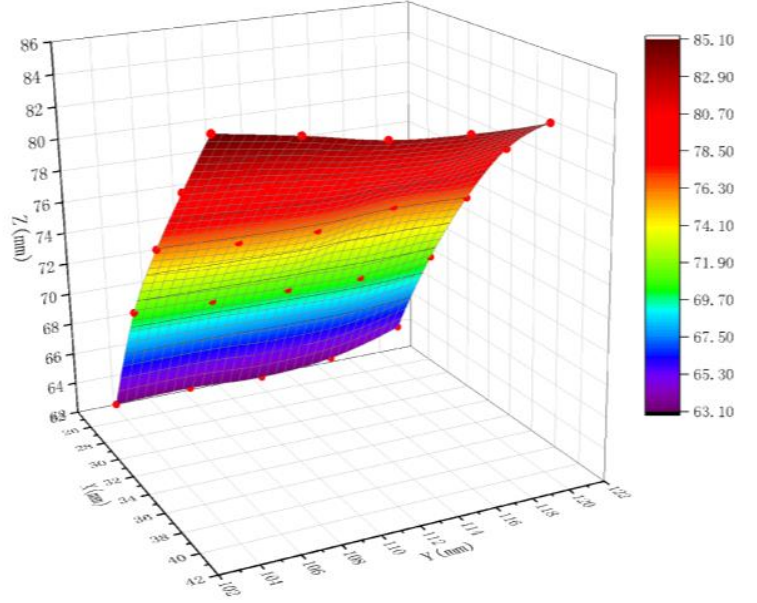

Figure 4.12 Linear displacement image of single branch chain

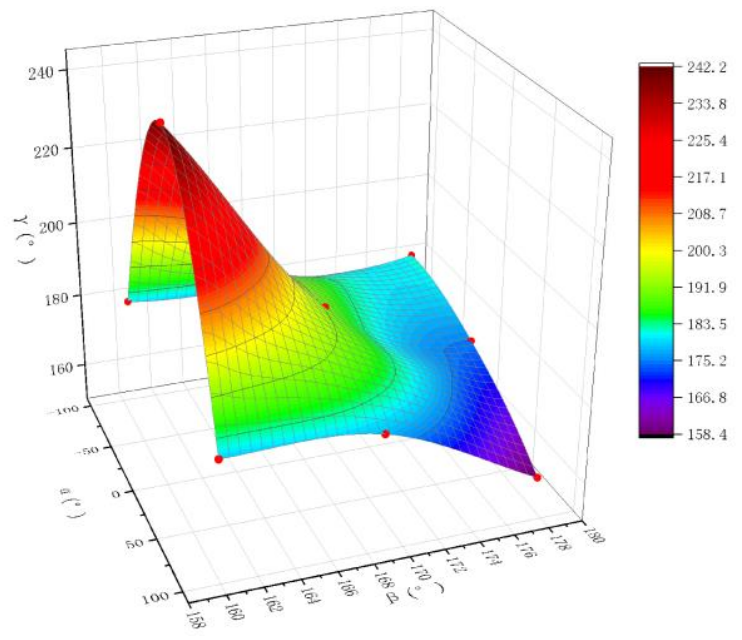

Figure 4.13 Euler angle of single branch chain movement

It can be seen from Figure 4.12 that the linear displacement of the mechanism changes smoothly within the reach of the working space, and there is no major sudden change. It shows that the mechanism moves smoothly and reliably without interference, and has certain spatial accessibility. It can be seen from Figure 4.13 that the movement surface of the mechanism within the reach of the working space gradually increases from one end to the central area, and forms a higher sudden change at the other end, which changes smoothly and has a good opening and closing degree.This indicates that the de- 
signed mechanism has a good range of spatial motion, can adapt to a relatively large posture and work space, and can meet the design requirements of ankle joint rehabilitation movement angle changes.

Given a driving joint with a unit velocity vector that can be along any direction in the joint space, constraints are added to the joint variables. According to the constraint conditions and the actual demand movement angle, the kinematics image of the corresponding surface in the working space of the mechanism is solved, and then the three-dimensional image of the driving input speed can be obtained. The output speed set of the upper platform corresponding to the corresponding driving speed can be obtained through the 4.3 .1 speed solving formula.

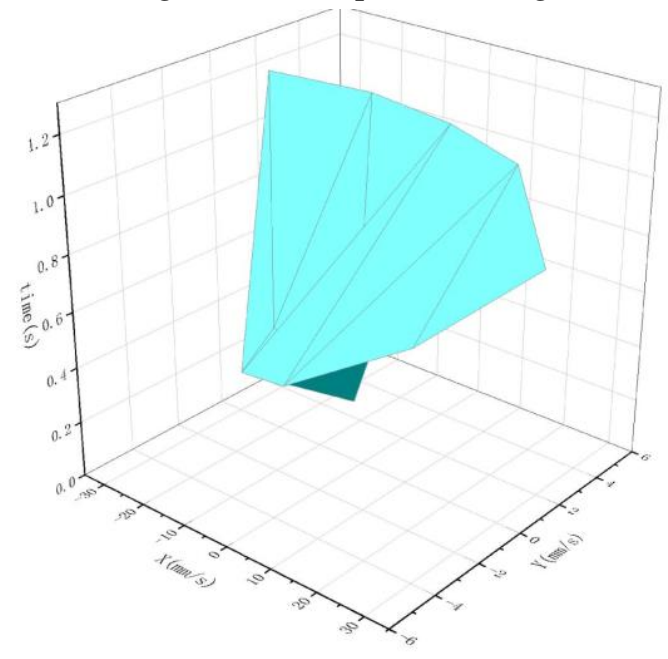

Figure 4.14 Speed change image

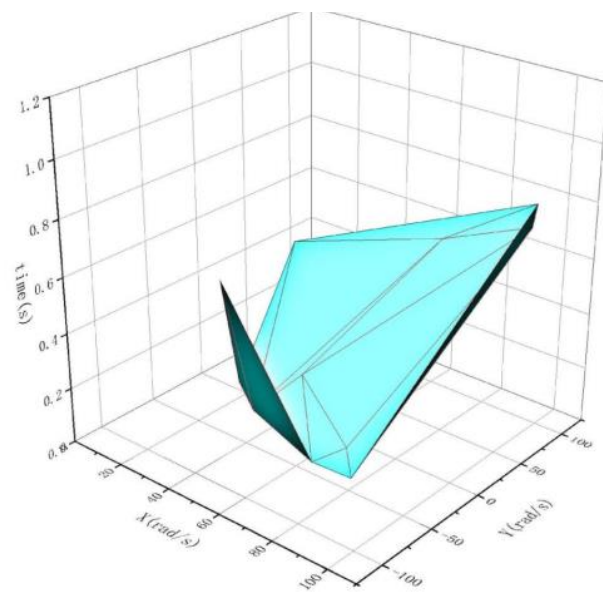

Figure 4.15 Angular velocity change image

It can be seen from Figure 4.11 that the speed $v_{x}$ and $v_{y}$ change with time, and the image surface is close to a plane. It shows that the speed is continuous, stable, and close to linear. However, there is a sudden bending change at the bottom, which indicates that the speed transmission capacity of the mechanism under the mechanism parameters is different. The speed transmission performance of the mechanism is similar, and the speed performance is better. It can be obtained from the figure 4.15 that the angle surface of the mechanism movement is close to the fan surface, and it can be concluded that the angular velocity changes smoothly during the movement. So as to ensure the feasibility of the actual application of the organization. When the mechanism has good speed characteristics, it will greatly help the ankle joint rehabilitation.

\section{Conclusions}


In this paper, a new type of ankle joint rehabilitation robot based on 4-UPUS parallel mechanism is proposed, and the overall structure design and rehabilitation process plan are established. A six-link kinematic model is established, and the kinematics diagram is obtained through calculation and simulation. The kinematic model of the parallel mechanism is constructed, and the three-dimensional figures such as the attitude angle and linear displacement of the reachable workspace are obtained. The research results show that the 4-UPUS parallel mechanism rehabilitation training robot has good space and flexibility, can provide patients with the needs of rehabilitation treatment at various angles, and can meet the maximum application in the field of ankle joint rehabilitation. It solves the problems of single movement and insufficient spatial flexibility of the current lower limb rehabilitation robot.

However, in the kinematics analysis, the velocity image appears abrupt change, indicating that the mechanism interferes in the movement, and further debugging and optimization of the mechanism is required. In this paper, only the overall structure work is done, and the content of control needs to be further studied. According to the requirements of ankle joint rehabilitation training, the control system of the ankle joint rehabilitation robot is designed to adapt to various rehabilitation strategies. The existing virtual reality technology can realize force feedback. Through the human-computer interaction interface, patients can observe gait motion images in real time, monitor their own rehabilitation status more intuitively, and increase the interest and practicability of patients' rehabilitation treatment. It will be the focus of the next research work to make it truly used in clinical practice for ankle rehabilitation.

Author Contributions: Conceptualization, A.H., Q.Y.and J.A.; methodology, A.H. and Q.Y.; software, A.H.; validation, A.H., Q.Y. and J.A.; formal analysis, A.H.; investigation, A.H.; resources, A.H.; data curation, A.H.; writing-original draft preparation, A.H.; writing-review and editing, A.H.; visualization, A.H.; supervision, X.D.; project administration, C.H.; funding acquisition, J.A.. All authors have read and agreed to the published version of the manuscript.

Funding: This study was funded by the "Science and Technology Research Project of Hubei Provincial Department of Education", approval number: B2019408

Data Availability Statement: Not applicable.

Conflflicts of Interest: The authors declare no conflflict of interest.

Abbreviations:

The following abbreviations are used in this manuscript:

UPUS U-Universal hinges,P-Prismatic pair, U-Universal hinges,S-Spherical pair

\section{References}

1. Hussain S ,Jamwal P K,Vliet P V, et al. Robot Assisted Ankle Neuro-Rehabilitation: State of the art and Future Challenges. Expert Review of Neurotherapeutics, 2021, 21(6).[CrossRef]

2. Shi D, Zhang W, Ding X, et al. Parametric generation of three-dimensional gait for robot-assisted rehabilitation. Biology Open, 2020, 9(3):bio.047332.[CrossRef]

3. Pinheiro C ,Magalhes N ,J Figueiredo, et al.Wearable Biofeedback Improves Human-Robot Compliance during Ankle-Foot Exoskeleton-Assisted Gait Training: A Pre-Post Controlled Study in Healthy Participants. Sensors, 2020, 20(20):5876.[CrossRef]

4. Zang W, Zang K, Shen G, et al. Position, Jacobian, decoupling and workspace analysis of a novel parallel manipulator with four pneumatic artificial muscles. Journal of the Brazilian Society of Mechanical Sciences and Engineering, 2019, 41(3).[CrossRef]

5. Zhou G, Kim J , Yong J C . Jacobian approach to the kinestatic analysis of a full vehicle model with application to cornering motion analysis. ARCHIVE Proceedings of the Institution of Mechanical Engineers Part C Journal of Mechanical Engineering Science , 2020:(vols 203-210):1989-1996.095440622091650.[CrossRef]

6. Li L , Fang Y, Wang L . Design of a family of multi-DOF drive systems for fewer limb parallel mechanisms. Mechanism and Machine Theory, 2020, 148:103802.[CrossRef] 
7. Rosyid A , El-Khasawneh B , Alazzam A . External Kinematic Calibration of Hybrid Kinematics Machine Utilizing Lower-DOF Planar Parallel Kinematics Mechanisms. International Journal of Precision Engineering and Manufacturing, 2019:1-21.[CrossRef]

8. K Almaghout, Tarvirdizadeh B , K Alipour, et al. Design and control of a lower limb rehabilitation robot considering undesirable torques of the patient's limb. Proceedings of the Institution of Mechanical Engineers Part $\mathrm{H}$ Journal of Engineering in Medicine, 2020, 234(4):095441192094784.[CrossRef]

9. Jamwal P K, Hussain S, Yun H T , et al. Musculoskeletal Model for Path Generation and Modification of an Ankle Rehabilitation Robot. IEEE Transactions on Human-Machine Systems, 2020, 50(5).[CrossRef]

10. Li J , Zuo S , Zhang L, et al. Mechanical Design and Performance Analysis of a Novel Parallel Robot for Ankle Rehabilitation. Journal of Mechanisms and Robotics, 2020, 12(5):1-36.[CrossRef]

11. Zhang L, Ji Li, Dong M , et al. Design and Workspace Analysis of a Parallel Ankle Rehabilitation Robot (PARR). Journal of Healthcare Engineering, 2019, 2019:1-10.[CrossRef]

12. Zhang M ,TC Davies, Effectiveness of robot-assisted therapy on ankle rehabilitation - a systematic review. Journal of NeuroEngineering and Rehabilitation, 2013, 10(1):30.[CrossRef]

13. Russo M , Ceccarelli M . Analysis of a Wearable Robotic System for Ankle Rehabilitation. Machines, 2020, 8(3):48.[CrossRef]

14. Jiang J , Li W, Lee K M . A Novel Pantographic Exoskeleton based Collocated Joint Design with Application for Early Stroke Rehabilitation. IEEE/ASME Transactions on Mechatronics, 2020, 25(4):1922 - 1932.[CrossRef]

15. Yin, $\mathrm{Q}, \mathrm{Hu}, \mathrm{A}, \mathrm{Li}, \mathrm{Q}$, et al. Compound lower limb vibration training rehabilitation robot. Concurrency Computat Pract Exper. 2021; 33:e6059.[CrossRef]

16. Wang Z, Zhang W, Ding X. Design and analysis of a novel mechanism with a two-DOF remote centre of motion. Mechanism and Machine Theory, 2020, 153:103990.[CrossRef]

17. Rosyid A , El-Khasawneh B . Identification of the Dynamic Parameters of a Parallel Kinematics Mechanism with Prismatic Joints by Considering Varying Friction. Applied Sciences, 2020, 10(14):4820.[CrossRef]

18. Shi X, Lu H, Chen Z. Design and Analysis of an Intelligent Toilet Wheelchair Based on Planar 2DOF Parallel Mechanism with Coupling Branch Chains. Sensors, 2021, 21(8):2677.[CrossRef]

19. Jia $Y$, Chen $X$. Dynamic response analysis for multi-degrees-of-freedom parallel mechanisms with various types of three-dimensional clearance joints. International Journal of Advanced Robotic Systems, 2021, 18(3):172988142110177.[CrossRef]

20. Kumar S , Nayak A, H Peters, et al. Kinematic Analysis of a Novel Parallel 2SPRR+1U Ankle Mechanism in Humanoid Robot. 2018.8:431-439.[CrossRef]

21. Shahid H., Prashant K J., Mergen H G., State-of-the-art robotic devices for ankle rehabilitation: Mechanism and control review. Proceedings of the Institution of Mechanical Engineers Part H Journal of Engineering in Medicine, 2017, 231(12):095441191773758.[CrossRef]

22. Liu X, Zhang J, Liu C , et al. Kinematics analysis and scale optimization of four degree of freedom generalized spherical parallel mechanism for ankle joint rehabilitation,Journal of biomedical engineering , 2021, 38(2):286-294.[CrossRef]

23. Zhi W., Liao, Ligang, et al. Screw theory based mathematical modeling and kinematic analysis of a novel ankle rehabilitation robot with a constrained 3-PSP mechanism topology. International Journal of Intelligent Robotics \& Applications, 2018.(2).351$360[$ CrossRef]

24. Wang C, Wang L, Wang T , et al. Research on an Ankle Joint Auxiliary Rehabilitation Robot with a Rigid-Flexible Hybrid Drive Based on a 2-S ' PS ' Mechanism. Applied Bionics and Biomechanics, , 2019(4):1-20.[CrossRef]

25. Wang D, Wang W, Guo Q , et al. Design and validation of a foot-ankle dynamic simulator with a 6-degree-of-freedom parallel mechanism. Proceedings of the Institution of Mechanical Engineers Part H Journal of Engineering in Medicine, 2020, 234(10):095441192093890.[CrossRef]

26. Choi W H , Takeda Y . Displacement Analysis and Design of a (2-RRU)-URR Parallel Mechanism Performing 2R1T Output Motion for Thumb Rehabilitation. Robotics, 2020, 9(3):67.[CrossRef]

27. Liu J , Fan X, Ding H . Investigation of a novel 2R1T parallel mechanism and construction of its variants. Robotica, 2021:1-15.[CrossRef]

28. Azar W A, Nazar P S . An optimized and chaotic intelligent system for a 3DOF rehabilitation robot for lower limbs based on neural network and genetic algorithm. Biomedical Signal Processing and Control, 2021, 69(10):102864.[CrossRef]

29. Dong M , Kong Y , Li J , et al. Kinematic Calibration of a Parallel 2-UPS/RRR Ankle Rehabilitation Robot. Journal of Healthcare Engineering, 2020, https://doi.org/10.1155/2020/3053629[CrossRef]

30. Wang H , Lin M , Jin Z, et al. A 4-DOF Workspace Lower Limb Rehabilitation Robot: Mechanism Design, Human Joint Analysis and Trajectory Planning. Applied Sciences, 2020, 10(13):4542.[CrossRef]

31. Zhang J, Jin Z, Feng H. Type synthesis of a 3-mixed-DOF protectable leg mechanism of a firefighting multi-legged robot based on GF set theory. Mechanism and Machine Theory, 2018, 130:567-584.[CrossRef]

32. Li Y, Zhang Y, Zhang L . A New Method for Type Synthesis of 2R1T and 2T1R 3-DOF Redundant Actuated Parallel Mechanisms with Closed Loop Units. Chinese Journal of Mechanical Engineering, 2020, 33(1).[CrossRef]

33. Zhao C, Chen Z, Song J , et al. Deformation analysis of a novel 3-DOF parallel spindle head in gravitational field. Mechanism and Machine Theory, 2020, 154:104036.[CrossRef]

34. Kumar S, Bongardt B , Simnofske M, et al. Design and Kinematic Analysis of the Novel Almost Spherical Parallel Mechanism Active Ankle. Journal of Intelligent \& Robotic Systems, 2019 .94(3).[CrossRef] 
35. Zhou C, Nikos T. On the Comprehensive Kinematics Analysis of a Humanoid Parallel Ankle Mechanism. Journal of Mechanisms and Robotics, 2018, 10(5).[CrossRef]

36. Yu S, Zhang J, Li W , et al. New Decoupled 2-Dof Parallel Mechanism with Fully Spherical Workspace. Journal of Physics: Conference Series, 2020, 1575(1):012104 (7pp).[CrossRef]

37. Li Y, Zheng H, Chen B , et al. Dynamic Modeling and Analysis of 5PSS/UPU Parallel Mechanism with Elastically Active Branched Chains. Chinese Journal of Mechanical Engineering: English Edition, 2020,33(3):12.[CrossRef]

38. Lu B , Cui G ,Guo Q .Static and Dynamic Analysis of a 2T3R Five-degree-of-freedom Parallel Mechanism. IOP Conference Series: Materials Science and Engineering, 2020, 751(1):012060 (6pp).[CrossRef]

39. Chen Z, Li M , Kong X, et al. Kinematics analysis of a novel 2R1T 3-PUU parallel mechanism with multiple rotation centers. Mechanism and Machine Theory, 2020, 152(2):103938.[CrossRef]

40. Cao Y, Zhang T, Zhao Y, et al. Performance analysis of 3-PPRU parallel mechanism with a completely/partially/non constant Jacobian matrix. Journal of Mechanical Science and Technology, 2020, 34(10):4263-4279. [CrossRef] 